\title{
Brain Organization and Computation
}

\author{
Andreas Schierwagen \\ Institute for Computer Science, Intelligent Systems Department, University of \\ Leipzig, Leipzig, Germany \\ schierwa@informatik.uni-leipzig.de \\ http://www.informatik.uni-leipzig.de/〜 schierwa
}

\begin{abstract}
Theories of how the brain computes can be differentiated in three general conceptions: the algorithmic approach, the neural information processing (neurocomputational) approach and the dynamical systems approach. The discussion of key features of brain organization (i.e. structure with function) demonstrates the self-organizing character of brain processes at the various spatio-temporal scales. It is argued that the features associated with the brain are in support of its description in terms of dynamical systems theory, and of a concept of computation to be developed further within this framework.
\end{abstract}

\section{Introduction}

The brain as the basis of cognitive functions such as a thinking, perception and acting has been fascinating scientists for a long time, and to understand its operational principles is one of the largest challenges to modern science.

Only recently, the functional architecture of the brain has gained attention from scientific camps which are traditionally rather distant from neuroscience, i.e. from computer and organization sciences. The reason is that information technology sees an explosion of complexity, forming the basis for both great expectations and worries while the latter come up since software technology is facing a complexity bottleneck [1. Thus various initiatives started to propagate novel paradigms of Unconventional Computing such as IBM's 'Autonomic Computing' 1, the 'Grand Challenges in Computing Research' in the UK 2 , and DFG's 'Organic Computing' (DFG = German Science Foundation) 3 .

According to current views, the brain is both a computing and organic entity. The research initiatives mentioned before see therefore the neurosciences as sources of concepts relevant for the new, unconventional computing paradigms envisioned. Hence, the formal concepts which were developed within the Theoretical Neuroscience to describe and understand the brain as an information processing system are of special relevance.

This paper is organized as follows. Section 2 reviews some key features of brain organization (i.e. structure with function). It is followed by Section 3

\footnotetext{
${ }^{1}$ http://www.research.ibm.com/autonomic

2 http://www.ukcrc.org.uk/grand_challenges

${ }^{3}$ http://www.organic-computing.org 

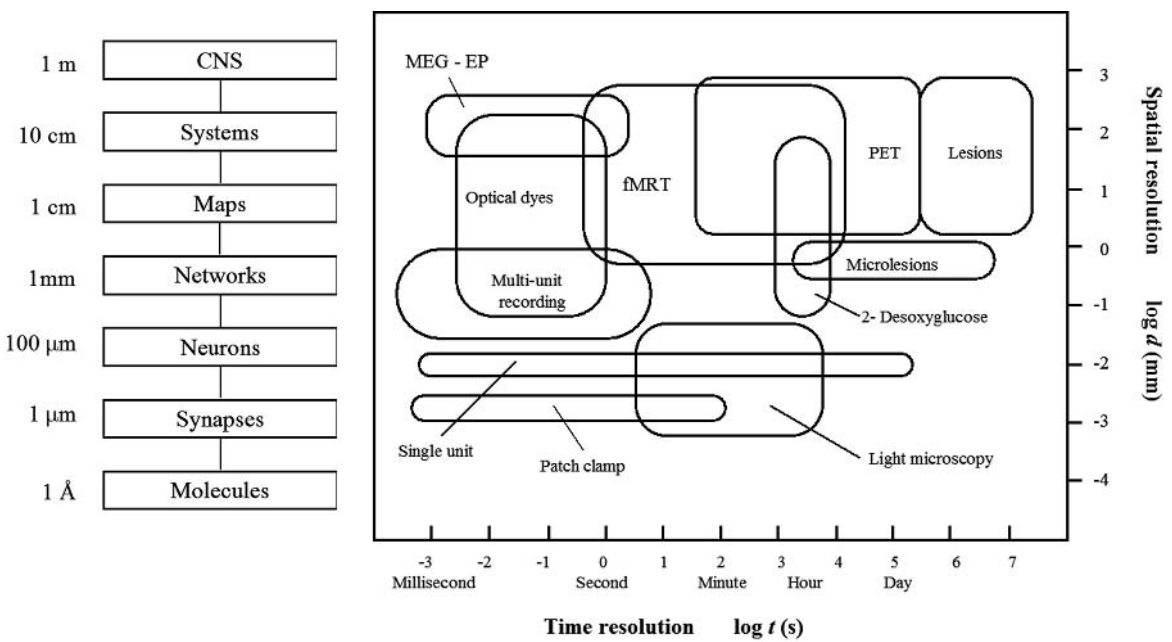

Fig. 1. Levels of brain organization and methods for its investigation. This figure relates the resolution in space and time of various methods for the study of brain function (right) to the scale at which neuronal structures can be identified (left). Adapted from 4. $\mathrm{MEG}=$ magnetoencephalography; $\mathrm{EP}=$ evoked potentials; $\mathrm{fMRT}=$ functional magnetic resonance tomography; PET=positron emission tomography.

which discusses the different computational approaches developed in Theoretical (Computational) Neuroscience. Questions raised there include the search for the computational unit, the concept of modularity and the development of dynamical systems approaches. We end in Section 4 with some conclusions concerning the needs of a theory of analog, emergent computation.

\section{Brain Organization and Methods for Investigation}

Neuroscientific research is practiced at very different levels extending from molecular biology of the cell up to the behavior of the organism. In the first line, naturally, the neuroscientific disciplines (Neuroanatomy, -physiology, -chemistry and -genetics) are involved, but also Psychology and Cognitive Science. Theoretical Neurobiology (with its subdivisions Computational Neuroscience and Neurocomputing), Physics and Mathematics provide theoretical contributions (e.g. [2[3]). The integration of the results gathered by the disciplines is expected to provide insights in the mechanisms on which the functions of neurons and neural networks are based, and in the long run in those of cognition. The well-grounded and efficient realization of this integration represents one of the greatest challenges of actual neurosciences. New techniques like patch clamp, multi-electrode recording, electroencephalogram (EEG) and imaging methods such as magnetoenzephalography (MEG), positron emission tomography (PET) and functional magnetic resonance tomography (fMRT, nuclear spin tomography) enable 
investigations on different system levels (Fig. 1), raising again the question of how to integrate conceptionally the results.

The human brain has on the average a mass of $1.4 \mathrm{~kg}$. According to different estimations it contains $10^{11}-10^{12}$ neurons which differ from other cells of the organism by the pronounced variability of their shapes and sizes (Fig. 22). The individual morphologic characteristics of the neurons are important determinants of neuronal function [56/789], and thus they affect the dynamic characteristics of the neural network, to which they belong, either directly, or by specifying the entire connectivity between the neurons. In neural systems the influences are mutual, so that in general also the global network dynamics affect the connectivity and the form of the individual constituent neurons [5[10].

A

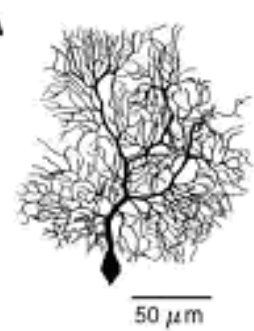

C

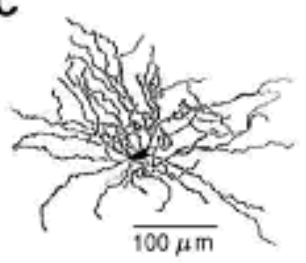

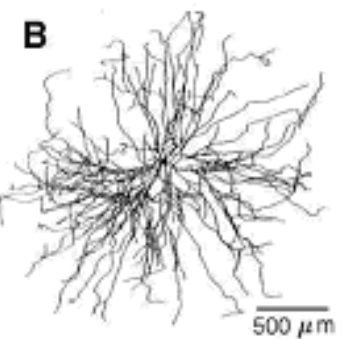

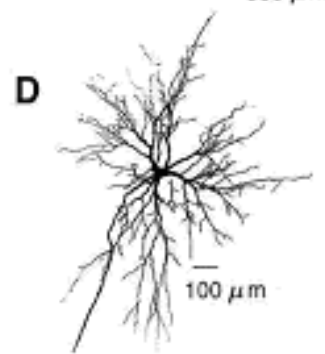

Fig. 2. Examples of dendritic neurons. Dendrites exhibit typical shapes which are used for classification of neurons. A. Purkinje cell from guinea pig cerebellum, B. amotoneuron from cat spinal chord, C. spiny neuron from rat neostriatum, D. Output neuron from cat superior colliculus. Figures A.-C. from [11, D. from 12].

The specific functions of the brain are essentially based on the interactions each of a large number of neurons by means of their synaptic connections. A mammalian neuron supports between $10^{4}$ and $10^{5}$ synapses whose majority is located on the dendrites. Estimations of the total number of synaptic connections in the human brain amount to $10^{15}$. Depending on the effect upon the successor neurons connections are classified as excitatory and inhibitory. The neurons of the cortex are usually assigned to two main categories: the pyramidal cells with a portion of ca. $85 \%$, and the stellate cells with ca. $15 \%$ [13. Pyramidal neurons often have long-range axons with excitatory synapses, and stellate cells with an only locally branched axon often act in an inhibitory manner. The 
activation status of the pyramidal neurons possibly encodes the relevant information, while the stellate cells raise the difference between center and surround by their inhibitory influence on the local environment, i.e. by lateral inhibition.

On the basis of distribution, density and size of the neuron somata the cortex can be divided in six layers (e.g. 14]). The cell bodies of the pyramidal cells particularly are in the layers III-V, and their apical dendrites extend into the upper layer I. The somata of the stellate cells are mainly in the middle layers IIIIV (see (Fig. 31). Efferent connections from the cortex to subcortical and other structures are formed by the axons of the pyramidal cells in layer V; afferences to the cortex mainly come from the thalamus.

Hubel and Wiesel's landmark studies [15/16] of the visual system have led to the assumption that information processing in the brain generally follows a hierarchical principle. Important for the conceptional view on the function of the brain is, however, that there is also a multitude of feedback connections or 'back projections', which e.g. in the geniculate body (CGL) by far outnumber the forward connections. Nearly all brain regions influence themselves by the existence of such closed signal loops [17. This also applies to the function of the individual neurons, which are involved in signal processing within an area or a subsystem of the brain. Further operational principles are divergence and convergence of the connections, i.e. a neuron and/or an area sends its signals to many others, and it also receives signals from many other neurons and/or areas. On the average, any two neurons in the cortex are connected by only one other neuron ('two degrees of separation', cf. [18]). This structurally caused functional proximity means in the language of information processing that the brain is characterized through massive parallelism.

\section{Computational Approaches}

Theories of how the brain functions as an informational system are in different ways related to the levels of brain organization. We can differentiate three general conceptions : the algorithmic approach, the neural information processing (neurocomputational) approach and the dynamical approach [19].

The algorithmic computation approach attempts to use the formal definition of computation, originally proposed by Turing [20] in order to understand neural computation. Although brains can be understood in some formal sense as Turing machines, it is now generally accepted that this reveals nothing at all of how the brain actually works [19. Thus, Turing's definition of computation cannot be straightly applied (e.g. [21]).

The neurocomputational approach was launched in 1988 by Sejnowski, Koch and Churchland [4. By stressing the architecture of the brain itself Computational Neuroscience was defined by the explicit research aim of "explaining how electrical and chemical signals are used in the brain to represent and process information". In this approach, computation is understood as any form of process in neuronal systems where information is transformed [22. The 'acid test' for this approach (not passed as yet) is to find a definition for transformation of 


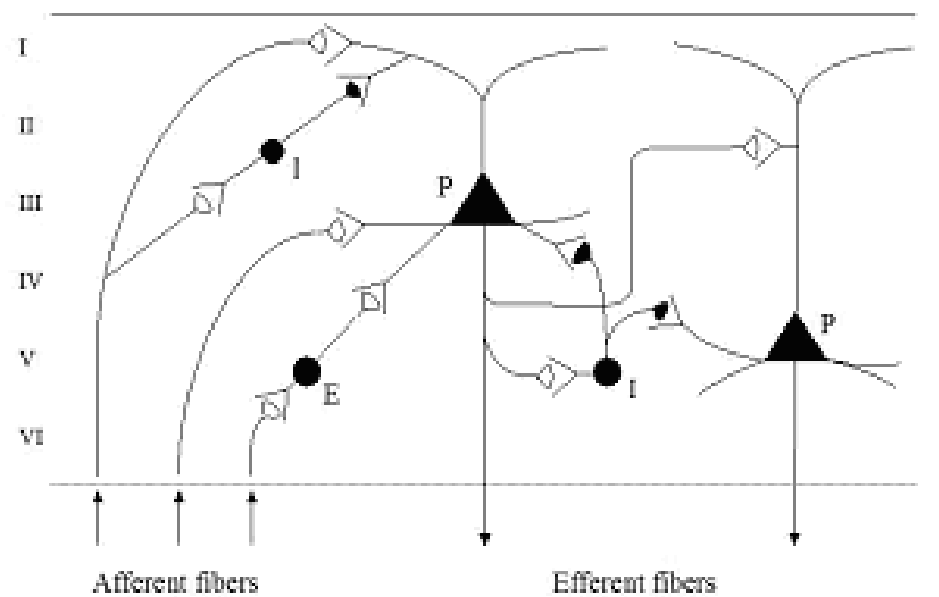

Fig. 3. Scheme of a neuronal circuit in the cerebral cortex. Pyramidal neurons (P black triangles) receive inputs (either directly via afferent fibers, or from local neurons), generate outputs, and interact with one another. Local neurons (black circles - various types of stellate cells) may be excitatory (E - empty synapse symbols) or inhibitory (I - black synapse symbols). Cortex layers are indicated on the left. Significant variations in cell density, dendritic architecture, and synaptic arrangement enable a vast number of computational possibilities.

information, such that not almost all natural systems count as computational information processors 2324 .

The dynamical approach rests on concepts and theories from the sciences (Mathematics, Physics, Chemistry and Biology), and particularly from (Nonlinear) Dynamical Systems Theory. It seeks to understand the brain in terms of analog, rate-dependent processes and physics style models. The brain is considered as a large and complex continuous-time (often also continuous-space) physical system that is described in terms of the dynamics of neural excitation and inhibition.

\subsection{Neurocomputational Concepts}

While current neurocomputational concepts are of great diversity, most of them are tightly linked to the algorithmic view. The algorithmic as well as the neurocomputational approach attempt to explain properties of the nervous system (e.g., object recognition) in terms of parts of the system (cardinal cells, or 'grandmother neurons'), in accordance with the decomposition principle of (linear) Systems Theory. Models of this kind seek to understand on a detailed level how synapses, single neurons, neural circuits and large populations process information. If the information processing capacity of the brain is compared in this way with that of an algorithmic computer, one is confronted with several problems. In the first line, the units of computation are to be determined. The identification of the computational elements, however, is highly controversial. 
As is generally known, McCulloch and Pitts in their now classical work 25] defined the neuron as the basic computational unit, since they believed it were the simplest nonlinear element.

Yet today it is obvious that (nonlinear) neuronal computation happens already at subcellular scales (dendritic subunits, synaptic sites), possibly even in supramolecular structures in dendrites. 26/27/28]. Correspondingly, e.g. synapses as computational units were analyzed in theoretical studies (e.g. [29]). But the problem of the computational unit at these scales remains open [30].

Computational units are assigned to supracellular scales, too. Based on ideas intimately related to the decomposability principle underlying the algorithmic approach, the principle of the modular organization of the brain has been formulated. According to this principle, the nervous system is composed of 'building blocks' of repetitive structures. The idea became known as the hypothesis of the columnar organization of the cerebral cortex; it was developed mainly after the works of Mountcastle, Hubel and Wiesel, and Szenthágothai (for reviews, see e.g. 31/32[33]).

Referring to and based on these works, the spectacular Blue Brain Project was started very recently. According to self-advertisement, the "Blue Brain project is the first comprehensive attempt to reverse-engineer the mammalian brain, in order to understand brain function and dysfunction through detailed simulations" [34. The central role in this project play 'cortical microcircuits' which have been suggested as modules computing basic functions. Indeed, impressive progress has been made in developing computational models for defined 'canonical' microcircuits, especially in the case of online computing on time-varying input streams (see [35] and references therein).

It should be noted, however, that the concept of columnar organization has been questioned by neurobiological experts. Reviewing new findings in different species and cortical areas, it was concluded that the notion of a basic uniformity in the neocortex, with respect to the density and types of neurons per column is not valid for all species [36. Other experts even more clearly state that it has been impossible to find a canonical microcircuit corresponding to the cortical column [37. These authors reason that although the column is an attractive idea both from neurobiological and computational point of view, it has failed as an unifying principle for understanding cortical function.

\subsection{Concepts from Dynamical Systems Theory}

Inconsistencies between neurobiological facts and theoretical concepts are not new in the history of Theoretical Neurobiology. In the case of the column concept they demonstrate that the decomposition principle is possibly not suitable to serve as exclusive guidance principle for the study of information processing in the brain. While the principle of decomposability has a great number of advantages, for example just modularity, many problems in neuroscience seem not decomposable this way. The reason is that brains (like all biological systems) are inherently complex. 
An appropriate framework for the description of the behavior of complex systems is represented by the attractor concept of nonlinear dynamical systems theory. Attractors may be informally defined as states of activity toward which a system settles (relaxes) over time. The activity in a neural system is described by a trajectory in the high-dimensional state space, say $R^{N}$ where $N$ is the number of neurons. Since this state (or phase) space is continuous, the neural system performs an analog computation [38. In this framework, a certain parameter setting (the initial condition) is interpreted as input, the attractor to which the system's state flows as the output, and the flow itself as the process of computation. The criteria of computational complexity developed for digital algorithms are not directly applicable to 'analog algorithms'. Appropriate criteria of 'dynamic complexity' have been suggested: the time of convergence to an attractor within defined error bounds, the degree of stability of the attractor, the pattern of convergence (asymptotic, or oscillatory), type of the attractor (static, periodic, chaotic, stochastic), etc. Important building blocks for a non-standard theory of computation in continuous space and time have been developed by Siegelmann [39] by relating the dynamical complexity of neural networks with usual computational complexity.

While standard artificial neural networks have only point attractors, dynamical systems theory easily handles also cases where the output is a limit cycle or a chaotic attractor. The respective systems, however, have not been considered in computational terms as yet. This holds also for the so-called active, excitable or reaction-diffusion media, of which continuous neural fields are instances (see [40]). These media - spatially extended continua - exhibit a variety of spatiotemporal phenomena. Circular waves, spiral waves, and localized mobile excitations ('bumps') are the most familiar examples. The challenge is to find out how these phenomena can be used to perform useful computations. Generally, data and results are given by spatial defects and information processing is implemented via spreading and interaction of phase or diffusive waves. In several studies it was shown that these media have real capabilities to solve problems of Computational and Cognitive Neuroscience (formation of working memory, preparation and control of saccadic eye movements, emergence of hallucinations under the influence of drugs or the like, 'near-to-death' experiences, for overview see e.g. [41/42] and the references therein) and Artificial Intelligence (navigation of autonomous agents, image processing and recognition, e.g. [43[44]).

\section{Conclusions}

During the last decade, useful insights on structural, functional and computational aspects of brain networks have been obtained employing network theory [45]46. From the many investigations in this area (see e.g. 47. for review) we know that the complexity of neural networks is due not only to their size (number of neurons) but also to the interaction of its connection topology and dynamics (the activity of the individual neurons), which gives rise to global states and emergent behaviors. 
Several attempts were made to substantiate the general idea of computational systems which acquire emergent capabilities during a process of self-organization. The holistic properties of self-organizing systems represent a central intricacy in this respect. There is no 'natural' way to decompose such a system. If a decomposition is made anyhow (e.g. based on anatomical information only), subsystems should at first have a certain behavioral potential (i.e. multi-functionality). Ideas of the unfolding of multi-functionality were subsumed by Shimizu [48] under the term relational system. Relational systems obtain their functional properties only during mutual interaction with the other elements of the system while on its part the interactions of the elements depend on the evolving properties of the elements. Thus, an iterative process takes place which is based on principles of self-reference and self-organization. The properties of the system as a whole emerge in such a way that it is able to cope with perturbations from the environment.

An attempt to formalize this concept was undertaken recently 49 using 'chaotic neuromodules'. The results obtained from applications to evolutionary robotics demonstrate the multi-functional properties of coupled chaotic neuromodules but also the limitations of the linear couplings used [50].

A general conclusion to be drawn is that a great deal of progress in Theoretical Neuroscience will depend on tools and concepts made available through the dynamical systems approach to computing. Steps to overcome the existing theoretical restrictions in this area are essential not only for solving the problems in the Neurosciences but also to reach the goals of Unconventional Computing.

\section{References}

1. Heylighen, F., Gershenson,C.: The meaning of self-organization in computing. IEEE Intelligent Systems (2003) 72-86

2. Schierwagen, A.: Real neurons and their circuitry: Implications for brain theory. iir-reporte, AdW der DDR, Eberswalde (1989) 17-20

3. Schierwagen, A.: Modelle der Neuroinformatik als Mittler zwischen neurobiologischen Fakten und Kognitionstheorien. In: Maaz, J. (ed.): Das sichtbare Denken Rodopi-Verlag, Amsterdam (1993) 131-152.

4. Senjowski, T.J., Koch C., Churchland, P.S.: Computational Neuroscience. Science 241 (1988) 1299-1306

5. Schierwagen, A.: Growth, structure and dynamics of real neurons: Model studies and experimental results. Biomed. Biochim. Acta 49 (1990) 709-722

6. Schierwagen, A., C. Claus: Dendritic morphology and signal delay in superior colliculus neurons. Neurocomputing 38-40(2001) 343-350.

7. Schierwagen, A., Van Pelt, J.: Synaptic input processing in complex neurons: A model study. In: Moreno-Diaz jr.,R. , Quesada-Arencibia,A., Rodriguez, J.-C. (eds.): CAST and Tools for Complexity in Biological, Physical and Engineering Systems - EUROCAST 2003. IUCTC, Las Palmas (2003) 221-225

8. Van Pelt, J., Schierwagen,A.: Morphological analysis and modeling of neuronal dendrites. Math. Biosciences 188 (2004) 147-155

9. Schierwagen, A., Alpár,A., Gärtner, U.: Scaling properties of pyramidal neurons in mice neocortex, Mathematical Biosciences (2006 doi:10.1016/j.mbs.2006.08.019 
10. Van Ooyen, A. (ed.): Modeling Neural Development. MIT Press, Cambridge MA (2003)

11. Segev, I.: Cable and Compartmental Models of Dendritic Trees. In: Bower, J.M., Beeman, D. (eds.): The Book of GENESIS: Exploring Realistic Neural Models with the GEneral NEural SImulation System. Telos/Springer-Verlag, Santa Clara, CA (1998)53-81

12. Schierwagen, A., Grantyn, R.: Quantitative morphological analysis of deep superior colliculus neurons stained intracellularly with HRP in the cat. J. Hirnforsch. 27 (1986) 611-623

13. Braitenberg, V., Schüz, A.: Anatomy of the Cortex: Statistics and Geometry. Springer, Berlin (1991)

14. Creutzfeld, O.: Cortex cerebri. Leistung, strukturelle und funktionelle Organisation der Hirnrinde. Springer-Verlag, Berlin etc. (1983)

15. Hubel D.H., Wiesel, T.N.: Receptive fields, binocular interaction and functional architecture in the cat's visual cortex. J. Physiol. 160 (1962) 106-154

16. Hubel D.H., Wiesel T.N.: Receptive fields and functional architecture of monkey striate cortex. J. Physiol. 195 (1968) 215-243

17. Sporns, O., Tononi, G., Edelman, G.M.: Theoretical neuroanatomy and the connectivity of the cerebral cortex. Behav. Brain Res. 135 (2002) 69-74

18. Watts, D.J., Strogatz, S.H.: Collective dynamics of "small-world networks. Nature 393 (1998) 440-442

19. Churchland, Patricia, and Grush, Rick (1999). Computation and the brain. In: Keil, F., Wilson, R.A. (eds.): The MIT Encyclopedia of Cognitive Sciences, MIT Press, Cambridge, MA (1999)155-158

20. Turing, A.M.: On computable numbers, with an application to the Entscheidungsproblem. Proc. Lond. Math. Soc. 42 (1936) 230-265

21. Mira, J., Delgado, A.E.: On how the computational paradigm can help us to model and interpret the neural function. Natural Computing (2006) DOI 10.1007/s11047006-9008-6

22. deCharms R.C., Zador, A.M.: Neural representation and the cortical code. Ann.l Rev. Neurosci. 23 (2000) 613-647

23. Searle, J. R.: Is the brain a digital computer? Proc. Amer. Philos. Assoc. 64 (1990) 21-37

24. Grush, R.: The semantic challenge to computational neuroscience. In: Machamer, P.K., Grush, R., McLaughlin, P. (eds.): Theory and method in the neurosciences. University of Pittsburgh Press, Pittsburg (2001)155-172

25. McCulloch WS, Pitts W: A logical calculus of the ideas immanent in nervous activity. Bull. Math. Biol. 52 (1943) 99-115

26. Euler, T., Denk, W.: Dendritic processing. Curr. Opin. Neurobiol. 11 (2001) 415422

27. Polsky, A., Mel, B.W., Schiller, J.: Computational subunits in thin dendrites of pyramidal cells. Nature Neurosci. 7 (2004) 621-627

28. London, M., Hausser, M.: Dendritic computation. Annu. Rev. Neurosci. 28 (2005) 503-532

29. Maass, W., Zador, A.M.: Synapses as Computational Units. Neural Computation 11 (1999) 903-917

30. Zador, A.M.: The basic unit of computation. Nature Neurosci. 3 (Suppl.) (2000) 1167

31. Hubel, D. H., Wiesel, T. N.: Functional architecture of macaque monkey cortex. Proc. R. Soc. London Ser. B 198 (1977) 1-59 
32. Mountcastle, V. B.: The columnar organization of the neocortex. Brain 120 (1997) 701-722

33. Szentágothai J. The modular architectonic principle of neural centers. Rev. Physiol. Bioche. Pharmacol. 98 (1983) 11-61

34. Markram, H.: The Blue Brain Project. Nature Rev. Neurosci. 7 (2006) 153-160

35. Maass, W., Markram, H.: Theory of the computational function of microcircuit dynamics. In S. Grillner and A. M. Graybiel, editors, The Interface between Neurons and Global Brain Function, Dahlem Workshop Report 93. MIT Press, Cambridge, MA (2006) 371-390

36. DeFelipe, J., Alonso-Nanclares, L., Arellano, J.I.: Microstructure of the neocortex: Comparative aspects. J. Neurocytol. 31 (2002) 299-316

37. Horton, J. C., Adams, D. L.: The cortical column: a structure without a function. Phil. Trans. R. Soc. B 360 (2005) 386-62

38. Siegelmann, H. T., Fishman, S.: Analog computation with dynamical systems. Physica D 120 (1998) 214-235

39. Siegelmann, H. T.: Neural Networks and Analog Computation: Beyond the Turing Limit. Birkhauser, Boston (1999)

40. Schierwagen, A., Werner, H.: Analog computations with mapped neural fields. In: Trappl, R.(ed.): Cybernetics and Systems '96, Austrian Society for Cybernetic Studies, Vienna (1996)1084-1089

41. Schierwagen, A., Werner, H.: Fast orienting movements to visual targets: Neural field model of dynamic gaze control. In: 6th European Symposium on Artificial Neural Networks - ESANN 98, D-facto publications Brussels (1998) 91-98

42. Wellner, J., Schierwagen, A.: Cellular-Automata-like Simulations of Dynamic Neural Fields. In: Holcombe, M., Paton,R.C. (eds.): Information Processing in Cells and Tissues, Plenum New York (1998) 295-304

43. Adamatzky, A.: Computing in Nonlinear Media: Make Waves, Study Collisions. In: Kelemen, J., Sosk, P. (eds.): ECAL 2001, LNAI 2159 (2001) 1-10

44. Sienko, T., Adamatzky, A., Rambidi, N.G., Conrad, M. (eds.): Molecular Computing. MIT Press, Cambridge, MA, London, England (2003)

45. Sporns, O., Chialvo, D.R., Kaiser, M., Hilgetag, C.C.: Organization, development and function of complex brain networks. Trends Cogn. Sci. 8 (2004) 418-25.

46. Buzsaki, G., Geisler, C., Henze, D.A., Wang, X.J.: Interneuron diversity series: circuit complexity and axon wiring economy of cortical interneurons. Trends Neurosci. 27 (2004) 186-193

47. Bassett, D.S., Bullmore, E.: Small-world brain networks, Neuroscientist 12 (2006) $512-523$

48. Shimizu, H.: Biological autonomy: the self-creation of constraints, Applied Mathematics and Computation, 56 (1993) 177-201

49. Pasemann, F.: Neuromodules: A dynamical systems approach to brain modelling. In: Herrmann, H. J., Wolf, D. E., Poppel, E. (eds.): Supercomputing in Brain Research: From Tomography to Neural Networks. World Scientific, Singapore (1995) 331-348

50. Hülse, M., Wischmann, S., Pasemann, F.: The role of non-linearity for evolved multifunctional robot behavior. In: Moreno, J.M., Madrenas, J., Cosp, J. (eds.): ICES 2005, LNCS 3637, (2005) 108-118 